\title{
Research and Manufacturing of Oval Gear Pair Applied in Rotor of a Roots Type Compressor
}

Nghiên cứu chế cặp bánh răng ô-van tựa elíp dẫn động rôto của một loại máy nén kiểu Roots

\author{
Nguyen Thanh Trung ${ }^{1,2}$, Nguyen Hoang Viet ${ }^{1}$, Nguyen Hong Thai ${ }^{2 *}$ \\ ${ }^{1}$ National Research Institute of Mechanical Engineering, Hanoi, Vietnam \\ ${ }^{2}$ Hanoi University of Science and Technology, Hanoi, Vietnam \\ ${ }^{*}$ Email: thai.nguyenhong@hust.edu.vn
}

\begin{abstract}
This study presents a method of shaping the tooth profile of an oval gear pair with the modified cycloid profile of the ellipse. The driving oval gear was shaped by a rack cutter with the profile of an improved cycloid, and the tooth profile of the driven oval gear was shaped by a shaper cutter to ensure that gear pair will mesh accurately with a function of transmission ratio. Mathematical model of the oval gear pair was established according to the theory of the non-circular gear with consideration to the conditions of the design parameters to avoid undercutting and tooth distribution of the oval gear. An improved pair of cycloid oval gears is designed and manufactured according to the results of the theoretical calculation. Furthermore, this gear pair is specifically applied to a Roots-type compressor to demonstrate its applicability in special cases where the traditional cylindrical gear pair cannot be replaced.
\end{abstract}

Keywords: Oval gears, modified cycloid profile, non-circular gear, rack cutter, shaper cutter.

Tóm tắt

Nghiên cứu này trình bày phương pháp tạo hình biên dạng răng của cặp bánh răng ô-van tựa elíp bằng đường xyclôít cải tiến của elíp. Trong đó, bánh răng chủ động được tạo hình bằng thanh răng sinh có biên dạng là đường xyclôít cải tiến, còn biên dạng răng của bánh răng ô-van bị động được tạo hình bằng bánh răng sinh chủ động để đảm bảo cặp bánh răng ăn khớp theo đúng hàm truyền. Phương trình biên dạng răng của cặp bánh răng ô-van tựa elíp được thiết lập theo lý thuyết ăn khớp của bánh răng không tròn và xét đến điều kiện của các thông số thiết kế nhằm tránh cắt lẹm chân răng và phân bố răng của bánh răng o van. Một cặp bánh răng ô-van biên dạng xyclôít cải tiến được thiết kế, chế tạo theo kết quả nghiên cứu lý thuyết của bài báo được ứng dụng cụ thể cho một máy nén kiểu Roots để minh chứng cho khả năng ứng dụng của cặp bánh răng này trong những trường hợp đặc biệt mà cặp bánh răng trụ tròn truyền thống không thể thay thế được.

Từ khóa: Bánh răng ô-van, biên dạng xyclôít cải tiến, bánh răng không tròn, thanh răng sinh, bánh răng sinh.

\section{1. Đặt vấn đề}

Bánh răng ô-van (BROV) là một trường hợp đặc biệt của bánh răng không tròn (BRKT) mặc dù đã được nghiên cứu phát triển từ thế kỷ thứ 14 [1]. Nhưng do thiết kế phức tạp và khó khăn trong chế tạo mà loại BROV chưa được ứng dụng phổ biến. Bắt đầu từ thế kỷ 21 với sự phát triển của các phương pháp gia công hiện đại, các máy công cụ gia công điều khiển số với độ chính xác cao, bánh răng ô-van bắt đầu được nghiên cứu và phát triển cho các kịch bản ứng dụng khác nhau. Guo và đồng nghiệp [2] đã ứng dụng $\mathrm{BROV}$ thiết kế cơ cấu máy cấy lúa. Tiếp theo đó Zhao $[3,4]$ đã cải tiến các BROV trong hệ BRKT kiểu hành tinh của máy cấy lúa của Guo để phát triển sang cấy các loại rau ăn lá khác. Nhóm của Ottaviano [5] đã nghiên cứu phát triển cặp bánh răng hình bầu dục trong máy bơm máu cơ học ứng dụng trong phẫu thuật tim. Nghiên cứu của $\mathrm{Xu}$ và Emura [6] đã ứng dụng các bánh răng elíp trong thiết kế cơ cấu đánh lái của rôbốt tự hành kiểu bánh xe v.v.. Cho đến hiện tại có thể nhận thấy có hai xu hướng thiết kế BROV cho các kịch bản ứng dụng khác nhau: (i) Thiết kế bánh răng elíp có trục quay ở tiêu cự của elíp $[7,8]$, (ii) Thiết kế BROV có trục quay tại tâm hình học của hình ô-van $[9,10]$. Còn tạo hình biên dạng răng có hai phương pháp chính đó là: (a) Tạo hình và gia công $\mathrm{BROV}$ bằng phương pháp bánh răng sinh [11], (b) Tạo hình biên dạng bánh răng bằng thanh răng sinh $[8,10]$. Ở phương pháp thứ hai này có hai dạng thanh răng sinh đó là (i) thanh răng sinh có biên dạng là cung tròn [7,9] để tạo biên dạng răng của BROV kiểu Novikov và (ii) biên dạng hình thang cân để tạo biên dạng răng của BROV có biên dạng thân khai truyền thống [7,12]. Tuy nhiên cả hai biên dạng trên đều gặp một nhược điểm đó là xuất hiện nhọn đỉnh răng ở vị trí bán trục lớn của BROV. Để khắc phục hiện tượng này Bair [12] đã dịch chỉnh dương thanh răng sinh. Tuy nhiên, giải pháp này lại gây ra khe hở cạnh răng trong

ISSN 2734-9381

https://doi.org/10.51316/jst.154.etsd.2021.31.5.5

Received: October 12, 2020; accepted: November 2, 2021 
quá trình ăn khớp và tăng khoảng cách trục của hai BROV dẫn đến sau khi dịch chỉnh sẽ có sai số hàm truyền, còn khi góc áp lực nhỏ lại xuất hiện cắt chân răng. Vì vậy, trường hợp này chỉ phù hợp với thiết kế bơm bánh răng. Còn khi thay biên dạng thanh răng hình thang bằng các cung tròn [9] thì ta đã cải thiện được hiện tượng nhọn đỉnh răng và cắt chân răng nhưng lại giảm chiều cao răng. Để khắc phục những nhược điểm trên trong nghiên cứu này chúng tôi sử dụng đường xyclôít cải tiến kiểu elíp để làm biên dạng răng của BROV dẫn động cho một loại máy nén kiểu Roots có hai rôto được dẫn động theo một hàm truyền, biến đổi theo thời gian như được mô tả trên Hình 1 .

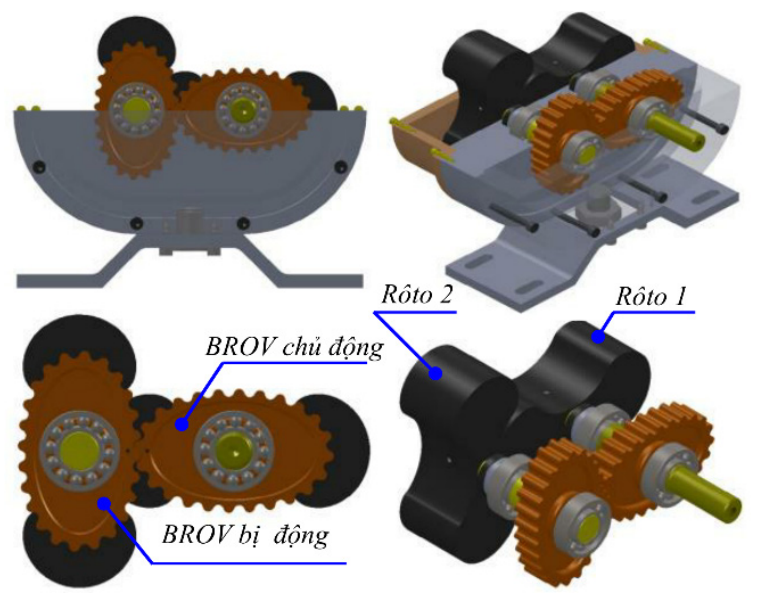

Hình 1. Máy nén kiểu Roots dẫn động bằng cặp BROV

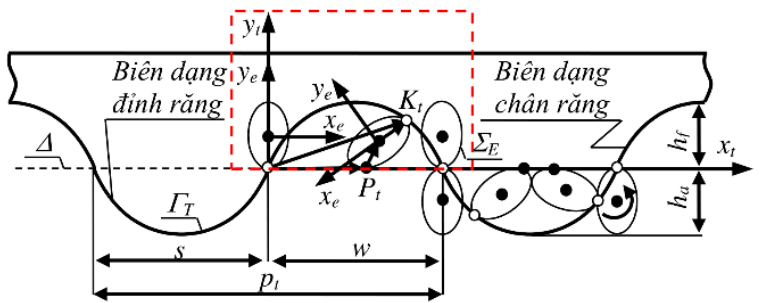

Hình 2. Tạo hình biên dạng răng

Để giải quyết vấn đề nêu trên nội dung bài báo trình bày: (1) Thiết lập phương trình biên dạng răng của $\mathrm{BROV}$ chủ động bằng phương pháp thanh răng sinh, để đảm bảo quá trình ăn khớp BROV bị động được tạo hình bằng bánh răng sinh (RROV chủ động); (2) xác định điều kiện của các thông số thiết kế hình dạng hình học $\mathrm{BROV}$ để tránh cắt lẹm chân răng và phân bố số răng; (3) Chế tạo thử nghiệm cặp BROV dẫn động cho một loại máy nén kiểu Roots mới nhằm minh chứng cho khả năng ứng dụng của $\mathrm{BROV}$ cho những trường hợp đặc biệt mà bánh răng trụ tròn truyền thống không thể đáp ứng.

\section{Phương trình biên dạng răng của thanh răng $\sinh$}

Phần biên dạng răng của thanh răng sinh $\Gamma_{T}$ là quỹ tích của một điểm $K$ cố định trên elíp sinh $\Sigma_{E}$, khi $\Sigma_{E}$ lăn không trượt với đường trung bình $\Delta$ của thanh răng sinh.
Như vậy, khi $\Sigma_{E}$ lăn không trượt phía trên $\Delta$ sẽ hình thành biên dạng chân răng, còn khi $\Sigma_{E}$ lăn không trượt phía dưới $\Delta$ sẽ hình thành biên dạng đỉnh răng như được mô tả trên Hình 2. Với nguyên lý hình thành biên dạng răng như trên, từ Hình 3 gọi $\vartheta_{t}\left\{O_{t} x_{t} y_{t}\right\}$ là hệ quy chiếu cố định gắn trên $\Delta, \vartheta_{e}\left\{O_{e} x_{e} y_{e}\right\}$ là hệ quy chiếu động gắn tại tâm $O_{e}$ của elíp sinh $\Sigma_{E}$ với $O_{e} y_{e}$ trùng với bán trục lớn.

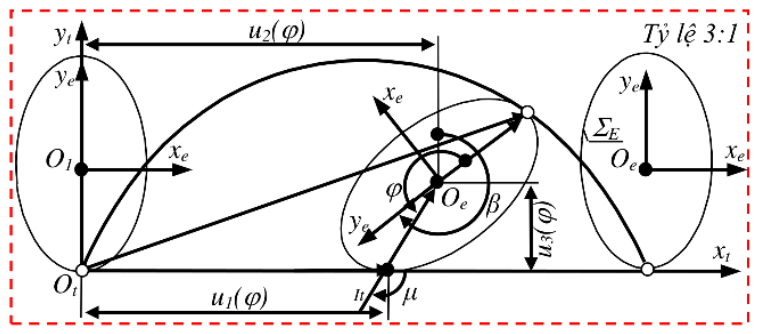

Hình 3. Tạo hình biên dạng răng

$I_{t}$ là điểm tiếp xúc giữa elíp sinh $\Sigma_{E}$ và $\Delta$ trong quá trình $\Sigma_{E}$ lăn không trượt với $\Delta$, khi đó phương trình biên dạng răng thanh răng sinh được cho bởi:

$$
\mathbf{r}_{\mathrm{K} t}=\mathbf{r}_{\mathrm{I}_{\mathrm{t}}}+\mathbf{r}_{\mathrm{O}_{\mathrm{e}}}+\mathbf{M r}_{\mathrm{K}}
$$

trong đó:

$$
\begin{aligned}
& \mathbf{r}_{\mathrm{K}_{\mathrm{t}}}=\left[\begin{array}{lll}
0 & -a & 0
\end{array}\right]^{T} ; \\
& \mathbf{r}_{\mathrm{O}_{\mathrm{e}}}=\left[\begin{array}{lll}
u_{2}(\phi) & u_{3}(\phi) & 0
\end{array}\right]^{T} ; \\
& \mathbf{r}_{\mathrm{I}_{\mathrm{t}}}=\left[\begin{array}{lll}
u_{1}(\phi) & 0 & 0
\end{array}\right]^{T} ; \\
&{ }^{\mathrm{t}} \mathbf{M}_{\mathrm{e}}=\left[\begin{array}{ccc}
\cos \beta(\phi) & \sin \beta(\phi) & 0 \\
-\sin \beta(\phi) & \cos \beta(\phi) & 0 \\
0 & 0 & 1
\end{array}\right] ;
\end{aligned}
$$

$u_{1}(\phi)=\int_{0}^{\phi} \sqrt{r_{E}(\phi)^{2}+\left(\frac{d r_{E}(\phi)}{d \phi}\right)^{2}} d \phi$ do điều kiện lăn không trượt của $\Sigma_{E}$ so với $\Delta ; r_{E}(\varphi)=a \sqrt{\frac{1-\varepsilon^{2}}{1-\varepsilon^{2} \cos ^{2} \varphi}}$ [14] là bán kính cực của $\Sigma_{E}$ với $\varepsilon=\frac{\sqrt{a^{2}-b^{2}}}{a}$ còn $a, b$ lần lượt là bán trục lớn và bán trục nhỏ của $\Sigma_{E}$; $u_{2}(\phi)=u_{1}(\phi)+r_{E}(\phi) \sin (\beta-\phi)$,

$u_{3}(\phi)=r_{E}(\phi) \cos (\beta-\phi)$

$\beta=\phi+\mu-\frac{\pi}{2}$ là góc quay của elíp sinh $\Sigma_{E}$ quanh tâm $O_{e} ; \mu=\arctan \left(\frac{r_{E}(\phi)}{d r_{E} / d \phi}\right)$ [8] là góc tiếp tuyến của elíp $\sinh \Sigma_{E}$ tại điểm tiếp xúc $P_{t}$; còn $\phi$ là góc cực của $\Sigma_{E}$.

Sau khi biến đổi và rút gọn (1) được viết lại: 


$$
\mathbf{r}_{\mathrm{K}_{\mathrm{t}}}=\left[\begin{array}{c}
x_{K_{t}} \\
y_{K_{t}} \\
0
\end{array}\right]=\left[\begin{array}{c}
u_{2}(\phi)-a \sin \beta \\
(-1)^{g} s_{3}(\varphi)-a \cos \beta \\
0
\end{array}\right]
$$

Trong phương trình (2) $g=0$ khi $\Gamma_{T}$ là phần biên dạng đỉnh răng của thanh răng sinh và $g=1$ khi $\Gamma_{T}$ là phần biên dạng chân răng của thanh răng sinh.

Từ nguyên lý hình thành $\Gamma_{T}$ các thông số thiết kế của thanh răng sinh được xác định:

Bước răng trên đường trung bình:

$$
p_{t}=t+w=2 \int_{0}^{2 \pi} \sqrt{r_{E}(\phi)^{2}+\left(\frac{d r_{E}(\phi)}{d \phi}\right)^{2}} d \phi
$$

trong đó $t, w$ lần lượt là chiều dày răng và chiều rộng rãnh răng $t=w$.

Chiều cao răng:

$$
h=h_{f}+h_{a}=4 a
$$

với $h_{f}, h_{a}$ lần lượt là chiều cao chân răng và chiều cao đỉnh răng $h_{f}=h_{a}=2 a$

Môđun được định nghĩa:

$$
m_{t}=\frac{p_{t}}{\pi}
$$

\section{Thiết kế cặp BROV dẫn động rôto của máy nén}

\section{kiểu Roots}

\subsection{Phưong trình đường lăn đối tiếp}

Từ Hình 4 gọi $a_{12}$ là khoảng cách trục của cặp $\mathrm{BROV} ; \Sigma_{1}, \Sigma_{2}$ lần lượt là đường lăn của $\mathrm{BROV}$ chủ động và bị động; $r_{l}\left(\phi_{1}\right)$ là bán kính cực của $\Sigma_{1}$ tại điểm $P_{1}$, còn $r_{2}\left(\phi_{2}\left(\phi_{1}\right)\right)$ là bán kính cực của $\Sigma_{2}$ tại điểm $P_{2} ; \phi_{1}, \phi_{2}\left(\phi_{1}\right)$ lần lượt là góc quay của $\mathrm{BROV}$ chủ động và bị động quay quanh tâm của từng bánh răng.

Theo [13] đối với cặp bánh răng dẫn động phải cùng hàm truyền với cặp rô-to của máy nén. Vì vậy, đường lăn $\Sigma_{l}$ của $\mathrm{BROV}_{1}$ phải có phương trình đường Ô-van tựa elíp như đối với cặp rôto:

$$
r_{1}\left(\phi_{1}\right)=2 a_{1} b_{1}\left(\left(a_{1}+b_{1}\right)-\left(a_{1}-b_{1}\right) \cos 2 \phi_{1}\right)^{-1}
$$

ở đây $a_{1}, b_{1}$ lần lượt là bán trục nhỏ và bán trục lớn của $\Sigma_{l}$ trên BROV và của rôto.

Do điều kiện $\Sigma_{1}, \Sigma_{2}$ lăn không trượt trên nhau tại tâm ăn khớp I phương trình đường lăn của BROV được cho bởi:

$$
\left\{\begin{array}{l}
r_{2}\left(\phi_{2}\left(\phi_{1}\right)\right)=a_{12}-2 a_{1} b_{1}\left(\left(a_{1}+b_{1}\right)-\left(a_{1}-b_{1}\right) \cos 2 \phi_{1}\right)^{-1} \\
\phi_{2}\left(\phi_{1}\right)=\int_{0}^{\phi_{1}}\left(\frac{2 a_{1} b_{1}\left(\left(a_{1}+b_{1}\right)-\left(a_{1}-b_{1}\right) \cos 2 \phi_{1}\right)^{-1}}{a_{12}-2 a_{1} b_{1}\left(\left(a_{1}+b_{1}\right)-\left(a_{1}-b_{1}\right) \cos 2 \phi_{1}\right)^{-1}}\right) d \phi_{1}
\end{array}\right.
$$

Khoảng cách trục $a_{12}$ của cặp bánh răng Ô-van được xác định bởi:

$$
\begin{aligned}
& f\left(n_{1}, a_{1}, b_{1}\right)= \\
& =\int_{0}^{\phi_{1}}\left(\frac{2 a_{1} b_{1}\left(\left(a_{1}+b_{1}\right)-\left(a_{1}-b_{1}\right) \cos 2 \phi_{1}\right)^{-1}}{a_{12}-2 a_{1} b_{1}\left(\left(a_{1}+b_{1}\right)-\left(a_{1}-b_{1}\right) \cos 2 \phi_{1}\right)^{-1}}\right) d \phi_{1}-2 \pi \\
& =0
\end{aligned}
$$

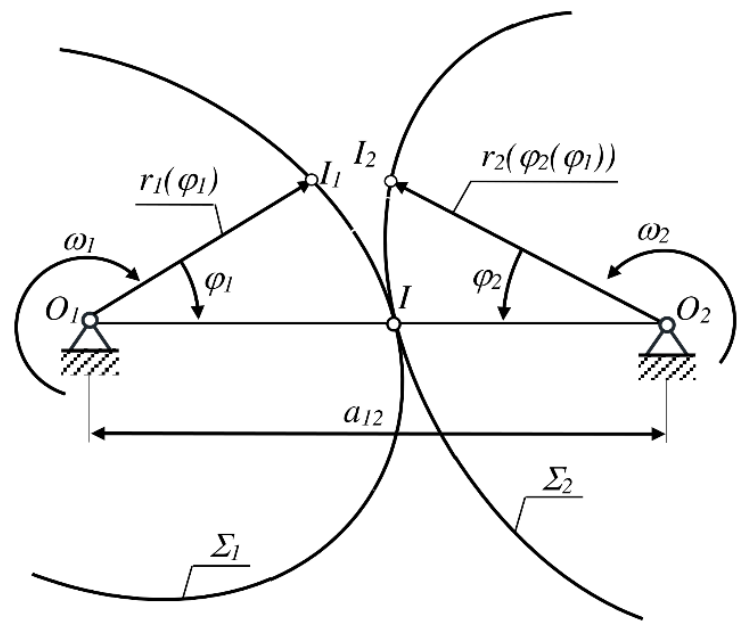

Hình 4. Cặp đường lăn đối tiếp

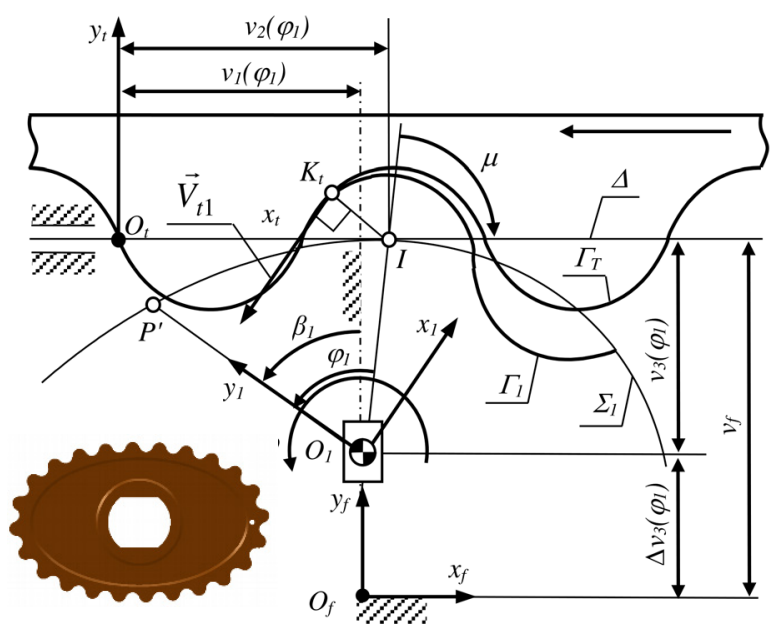

Hình 5. Tạo hình biên dạng răng

\subsection{Tạo hình biên dạng răng của bánh răng ô-van chủ động bằng thanh răng sinh}

3.2.1. Thiết lập phưong trình biên dạng răng của bánh răng $\hat{O}$-van chủ động

Để thiết lập phương trình biên dạng răng $\Gamma_{1}$ của $\mathrm{BROV}_{1}$, ta xét hệ quy chiếu cố định $\vartheta_{f}\left\{O_{f} x_{f} y_{f}\right\}$ gắn liền với giá và gọi $\vartheta_{1}\left\{O_{1} x_{1} y_{1}\right\}$ là hệ quy chiếu động gắn trên $\mathrm{BROV}_{1}$ như được mô tả trên Hình 5 .

Chuyển tọa độ điểm $K_{T}$ bất kỳ trên $\Gamma_{T}$ của thanh răng sinh trong hệ quy chiếu $\vartheta_{t}\left\{O_{t} x_{t} y_{t}\right\}$ về hệ quy 
chiếu $\vartheta_{1}\left\{O_{1} x_{1} y_{1}\right\}$ của $\mathrm{BROV}_{1}$ ta có phương trình biên dạng răng của $\mathrm{BROV}_{1}$ :

$$
\mathbf{r}_{K_{1}}=\mathbf{M}_{1 f} \mathbf{M}_{f t} \mathbf{r}_{K_{t}}
$$

trong đó

$\mathbf{M}_{1 f}=\left[\begin{array}{ccc}\cos \beta_{1}\left(\phi_{1}\right) & \sin \beta_{1}\left(\phi_{1}\right) & \Delta v_{3}\left(\phi_{1}\right) \sin \beta_{1}\left(\phi_{1}\right) \\ -\sin \beta_{1}\left(\phi_{1}\right) & \cos \beta_{1}\left(\phi_{1}\right) & \Delta v_{3}\left(\phi_{1}\right) \cos \beta_{1}\left(\phi_{1}\right) \\ 0 & 0 & 1\end{array}\right]$,

$\mathbf{M}_{f t}=\left[\begin{array}{ccc}1 & 0 & v_{2}\left(\phi_{1}\right) \\ 0 & 1 & v_{f} \\ 0 & 0 & 1\end{array}\right], \quad \mathbf{r}_{K_{t}}$ được xác định từ (8),

$\Delta v_{3}\left(\phi_{1}\right)=v_{f}-v_{3}\left(\phi_{1}\right)$ là khoảng dịch chuyển của tâm quay $O_{l}$ theo phương $y_{f}$ với $v_{f}$ là khoảng cách cố định từ $O_{f} x_{f}$ đến $O_{t} x_{t} ; v_{3}\left(\phi_{1}\right)=r_{1}\left(\phi_{1}\right) \cos \left(\beta_{1}-\phi_{1}\right)$ là khoảng dịch chuyển của tâm $O_{I}$ của BROV 1 theo phương $y_{f}$ khi điểm $I$ trên $\Sigma_{l}$ dịch chuyển đến vị trí $I^{\prime}$, $v_{2}\left(\phi_{1}\right)=v_{1}\left(\phi_{1}\right)+r_{1}\left(\phi_{1}\right) \sin \left(\beta_{1}-\phi_{1}\right)$ là khoảng tịnh tiến theo phương $x_{f}$ của thanh răng; $\beta_{1}=\phi_{1}+\mu_{1}-\frac{\pi}{2}$ là góc xoay của hệ quy chiếu $\vartheta_{1}\left\{O_{1} x_{1} y_{1}\right\}$ so với hệ quy chiếu $\vartheta_{f}\left\{O_{f} x_{f} y_{f}\right\}$ trong chuyển động tạo hình với $\mu_{1}=\arctan \left(\frac{r_{1}\left(\phi_{1}\right)}{d r_{1} / d \phi_{i}}\right)$ là góc tiếp tuyến tại $I$.

Khai triển (8) phương trình biên dạng răng $\Gamma_{l}$ của $\mathrm{BROV}_{1}$ được viết lại:

$\mathbf{r}_{K_{1}}=$

$\left[\begin{array}{l}\left(v_{2}\left(\phi_{1}\right)-x_{K_{t}}(\phi)\right) \sin \beta_{1}\left(\phi_{1}\right)+\left(v_{3}\left(\phi_{1}\right)+y_{K_{t}}(\phi)\right) \cos \beta_{1}\left(\phi_{1}\right) \\ \left(v_{2}\left(\phi_{1}\right)+y_{K_{1}}(\phi)\right) \sin \beta_{1}\left(\phi_{1}\right)-\left(v_{3}\left(\phi_{1}\right)-x_{K_{t}}(\phi)\right) \cos \beta_{1}\left(\phi_{1}\right)\end{array}\right]$

Mối quan hệ giữa hai thông số $\phi_{1}$ và $\phi$ được xác định thông qua điều kiện đối tiếp [15]:

$$
\mathbf{n} \mathbf{V}_{t 1}=0
$$

trong đó $\mathbf{n}$ là véc tơ pháp tuyến chung của cặp biên dạng đối tiếp $\left(\Gamma_{T}, \Gamma_{l}\right)$ tại $K$, còn $\mathbf{V}_{t 1}$ là vận tốc trượt tương đối giữa hai biên dạng $\left(\Gamma_{T}, \Gamma_{l}\right)$ tại điểm ăn khớp $K$. Véc tơ $\mathbf{n}$ được cho bởi:

$$
\mathbf{n}=\frac{\partial \mathbf{r}_{K_{t}}(\phi)}{\partial \phi} \times \mathbf{k}
$$

với $\mathbf{k}=\left[\begin{array}{lll}0 & 0 & 1\end{array}\right]^{T}$, còn $\mathbf{V}_{t 1}$ được cho bởi:

$$
\mathbf{V}_{t 1}=\omega_{t} \times{ }^{t} \mathbf{r}_{P K}
$$

trong đó $\omega_{t}=\omega_{t} \mathbf{k}_{t}$ là vận tốc góc của BROV 1 so với hệ quy chiếu $\vartheta_{t}\left\{O_{t} x_{t} y_{t}\right\}$ của thanh răng, còn ${ }^{\mathrm{t}} \mathbf{r}_{P K}=\left(u_{1}(\phi)-x_{K_{t}}\right) \mathbf{i}_{t}+y_{K_{t}} \mathbf{j}_{t}$.

Sau khi biến đổi $\mathbf{V}_{t 1}$ được cho bởi:

$$
\mathbf{V}_{t 1}=\omega\left[\begin{array}{c}
-y_{K_{t}}(\phi) \\
v_{1}\left(\phi_{1}\right)-x_{K_{t}}(\phi)
\end{array}\right]
$$

Thay $(13,14)$ vào công thức $(12)$ và rút gọn ta có: $f\left(\phi, \phi_{1}\right)=y_{K_{R}}^{\prime}(\phi) y_{K_{R}}(\phi)+x_{K_{R}}^{\prime}(\phi)\left(v_{1}\left(\phi_{1}\right)-x_{K_{R}}(\phi)\right)=0$

Giải (14) ta xác định được mối quan hệ giữa $\phi_{1}$ và $\phi$ của BROV 1 với thanh răng.

\subsubsection{Phân bố răng và điều kiện tránh cắt lẹm chân} răng của BROV chủ động

Do điều kiện lăn không trượt của $\Delta$ và $\Sigma_{1}$ nên bước răng $p_{1}$ trên vòng chia $\mathrm{BROV} 1$ bằng bước răng $p_{t}$ trên đường chia thanh răng sinh:

$$
p_{1}=p_{t}=t_{1}+w_{1}=t+w=2 C_{E}
$$

Khi đó số răng của BROV 1 được cho bởi:

$$
z_{1}=\frac{C_{\Sigma_{1}}}{p_{1}}
$$

trong đó $C_{1}=\int_{0}^{2 \pi} \sqrt{r_{1}\left(\phi_{1}\right)^{2}+\left(\frac{d r_{1}\left(\phi_{1}\right)}{d \phi_{1}}\right)^{2}} d \phi_{1}$ là chu vi của đường lăn $\Sigma_{1}$.

\subsection{3. Điều kiện tránh cắt lẹm chân răng của $B R O V$ chủ động}

Trong quá trình tạo hình biên dạng răng của $\mathrm{BROV}_{1}$ bằng thanh răng sinh, thanh răng không cắt lẹm vào chân răng của $\mathrm{BROV} 1$ thì phương trình biên dạng răng của thanh răng sinh phải thỏa mãn :

$$
\left\{\begin{array}{l}
\Delta_{1}=\left|\begin{array}{cc}
\frac{d x_{K_{t}}(\phi)}{d \phi} & -V_{t 1 x} \\
\frac{\partial f(\phi)}{\partial \phi} & \frac{\partial f\left(\phi_{1}\right)}{\partial \phi_{1}} \frac{d \phi_{1}}{d t}
\end{array}\right| \neq 0 \\
\Delta_{2}=\left|\begin{array}{cc}
\frac{d y_{K_{t}}(\phi)}{d \phi} & -V_{t 1 y} \\
\frac{\partial f(\phi)}{\partial \phi} & \frac{\partial f\left(\phi_{1}\right)}{\partial \phi_{1}} \frac{d \phi_{1}}{d t}
\end{array}\right| \neq 0
\end{array}\right.
$$

với $V_{t 1 x}, V_{t 1 y}$ là các thành phần của vận tốc trượt trên biên dạng thanh răng $\Gamma_{T}$ tại điểm tạo hình $K_{t}$ được xác định từ phương trình (13). 


\subsection{Tạo hình biên dạng răng của BROV bị động bằng bánh răng sinh}

3.3.1. Thiết lập phwơng trình biên dạng răng của BROV bị động

Để thiết lập phương trình biên dạng răng của bánh BROV bị động, xét trong hệ quy chiếu cố định $\vartheta_{f}\left\{O_{f} x_{f} y_{f}\right\}$ và gọi $\vartheta_{2}\left\{O_{2} x_{2} y_{2}\right\}$ là hệ quy chiếu động gắn trên $\mathrm{BROV} 2$ như được mô tả trên Hình 6 .

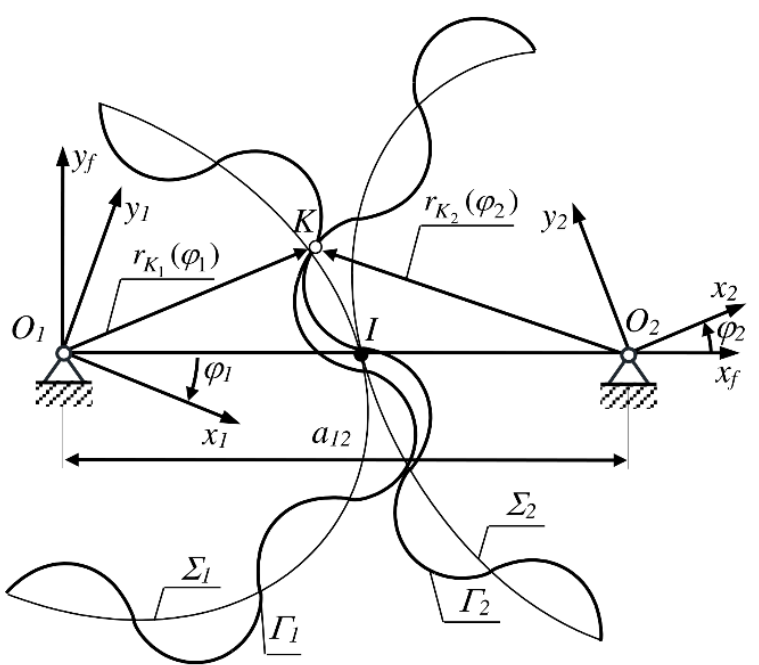

Hình 6. Tạo hình biên dạng răng của $\mathrm{BROV}_{2}$ bị động bằng bánh răng sinh

Chuyển tọa độ điểm $K_{l} \in \Gamma_{1}$ của $\mathrm{BROV}_{1}$ trong hệ quy chiếu $\vartheta_{1}\left\{O_{1} x_{1} y_{1}\right\}$ về hệ quy chiếu $\vartheta_{2}\left\{O_{2} x_{2} y_{2}\right\}$ của $\mathrm{BROV}_{2}$ bị động ta có phương trình biên dạng răng của $\mathrm{BROV}_{2}$

$$
\mathbf{r}_{K_{2}}=\mathbf{M}_{2 f} \mathbf{M}_{f 1} \mathbf{r}_{K_{1}}
$$

trong đó $\mathbf{M}_{2 f}=\left[\begin{array}{ccc}\cos \phi_{1} & \sin \phi_{1} & 0 \\ -\sin \phi_{1} & \cos \phi_{1} & 0 \\ 0 & 0 & 1\end{array}\right]$,

$\mathbf{M}_{f 1}=\left[\begin{array}{ccc}\cos \phi_{2}\left(\phi_{1}\right) & \sin \phi_{2}\left(\phi_{1}\right) & a_{12} \\ -\sin \phi_{2}\left(\phi_{1}\right) & \cos \phi_{2}\left(\phi_{1}\right) & 0 \\ 0 & 0 & 1\end{array}\right]$, còn $\mathbf{r}_{K_{1}}$ được

xác định từ (10).

Sau khi khai triển (19) phương trình biên dạng răng $\Gamma_{2}$ của $\mathrm{BRKT}_{2}$ được viết lại:

$$
\begin{aligned}
& \mathbf{r}_{K_{2}}= \\
& {\left[\begin{array}{c}
x_{K_{1}}\left(\phi_{1}\right) \cos \left(\phi_{1}+\phi_{2}\right)+y_{K_{1}}\left(\phi_{1}\right) \sin \left(\phi_{1}+\phi_{2}\right)+a_{12} \cos \phi_{1} \\
-x_{K_{1}}\left(\phi_{1}\right) \sin \left(\phi_{1}+\phi_{2}\right)+y_{K_{1}}\left(\phi_{1}\right) \cos \left(\phi_{1}+\phi_{2}\right)+a_{12} \sin \phi_{1}
\end{array}\right]}
\end{aligned}
$$

Trong (20) mối quan hệ $\phi_{2}\left(\phi_{1}\right)$ giữa $\phi_{2}$ và $\phi_{1}$ được xác định bởi (7).

\subsubsection{Thông số thiết kế của BROV bị động}

Do điều kiện lăn không trượt của $\Sigma_{1}$ và $\Sigma_{2}$ nên bước răng $p_{2}$ trên vòng chia $\mathrm{BROV}_{2}$ phải bằng bước răng $p_{I}$ trên vòng chia $\mathrm{BROV}_{1}$ :

$$
p_{2}=p_{1}=t_{2}+w_{2}=t_{1}+w_{1}=2 C_{E}
$$
bằng:

Do đó số răng $z_{1}$ của $\mathrm{BROV}_{1}$ và $\mathrm{BROV}_{2}$ phải

$$
z_{2}=n_{1} z_{1}
$$

\subsection{3. Điều kiện tránh cắt lẹm chân răng của BROV bị động}

Theo [8] để trong quá trình tạo hình biên dạng của bánh răng sinh không cắt lẹm vào chân răng của bánh răng được tạo hình thì phương trình biên dạng răng phải thỏa mãn:

$$
\left\{\begin{array}{l}
\Delta_{3}=\left|\begin{array}{cc}
\frac{d x_{K_{1}}\left(\phi_{1}\right)}{d \phi_{1}} & -V_{12 x} \\
\frac{\partial f(\phi)}{\partial \phi} & \frac{\partial f\left(\phi_{1}\right)}{\partial \phi_{1}} \frac{d \phi_{1}}{d t}
\end{array}\right| \neq 0 \\
\Delta_{4}=\left|\begin{array}{cc}
\frac{d y_{K_{1}}\left(\phi_{1}\right)}{d \phi_{1}} & -V_{12 y} \\
\frac{\partial f_{1}(\phi)}{\partial \phi} & \frac{\partial f\left(\phi_{1}\right)}{\partial \phi_{1}} \frac{d \phi_{1}}{d t}
\end{array}\right| \neq 0
\end{array}\right.
$$

với $V_{12 x}, V_{12 y}$ là các thành phần của vận tốc trượt $\mathbf{V}_{12}$ trên biên dạng thanh răng $\Gamma_{l}$ tại điểm tạo hình $K_{l} . \mathbf{V}_{12}$ được cho bởi:

$$
\mathbf{V}_{12}=\left({ }^{(1)} \boldsymbol{\omega}_{1}-{ }^{(2)} \boldsymbol{\omega}_{1}\right) \times \mathbf{r}_{K_{1}}-\left(\mathbf{a}_{12} \times{ }^{(2)} \boldsymbol{\omega}_{1}\right)
$$

Sau khi khai triển công thức (23) và biến đổi thì:

$$
\mathbf{V}_{12}=\omega_{1}\left[\begin{array}{l}
\left(1+i_{21}\left(\phi_{1}\right)\right) y_{K_{1}}(\theta)-a_{12} i_{21}\left(\phi_{1}\right) \sin \phi_{1} \\
\left(1+i_{21}\left(\phi_{1}\right)\right) x_{K_{1}}(\theta)+a_{12} i_{21}\left(\phi_{1}\right) \cos \phi_{1}
\end{array}\right]
$$

Với

$$
i_{21}\left(\phi_{1}\right)=\left(\frac{2 a_{1} b_{1}\left(\left(a_{1}+b_{1}\right)-\left(a_{1}-b_{1}\right) \cos 2 \phi_{1}\right)^{-1}}{a_{12}-2 a_{1} b_{1}\left(\left(a_{1}+b_{1}\right)-\left(a_{1}-b_{1}\right) \cos 2 \phi_{1}\right)^{-1}}\right)
$$

\section{Thiết kế và chế tạo cặp BROV dẫn động máy nén}

Theo [13] rôto của máy nén kiểu Roots được hình thành theo nguyên lý cặp bánh răng xyclôít như được mô tả trên Hình 7 trong đó cặp đường lăn $\left(\Sigma_{1}, \Sigma_{2}\right)$ được hình thành từ đường Ô-van tựa elíp cho bởi (6).

Từ Hình 7 với thông số thiết kế của máy nén, đường lăn Ô-van tựa elíp $\Sigma_{n 1}$ của rôto 1 có thông số kích thước: $a_{n 1}=50(\mathrm{~mm}), b_{n 1}=30(\mathrm{~mm})$ và khoảng cách trục $a_{12}=80(\mathrm{~mm})$, đường tròn sinh $\Sigma_{\mathrm{CS}}$ biên dạng rôto có bán kính $r=10,29(\mathrm{~mm})$ và mối quan hệ giữa góc quay của rôto ${ }_{2}$ so với rôto ${ }_{1}$ được mô tả trên Hình 8 . 


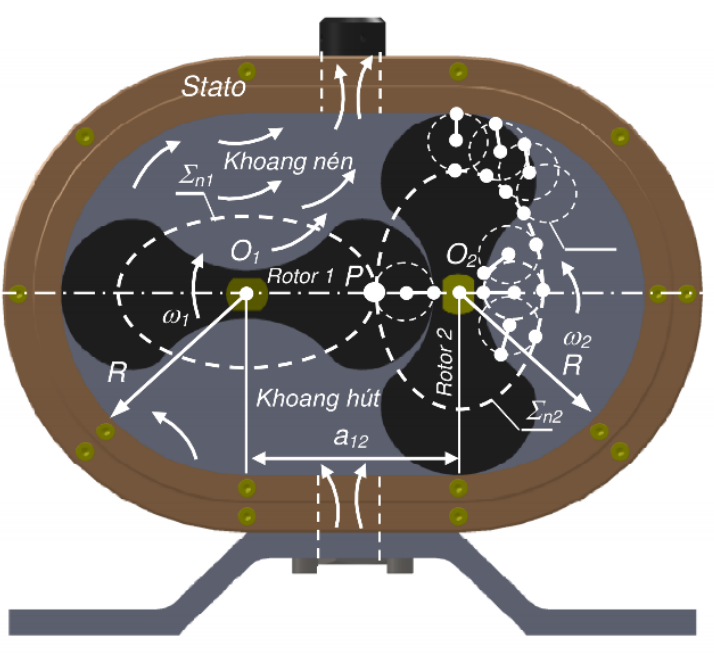

Hình 7. Nguyên lý hình thành rô-to của máy nén kiểu Roots

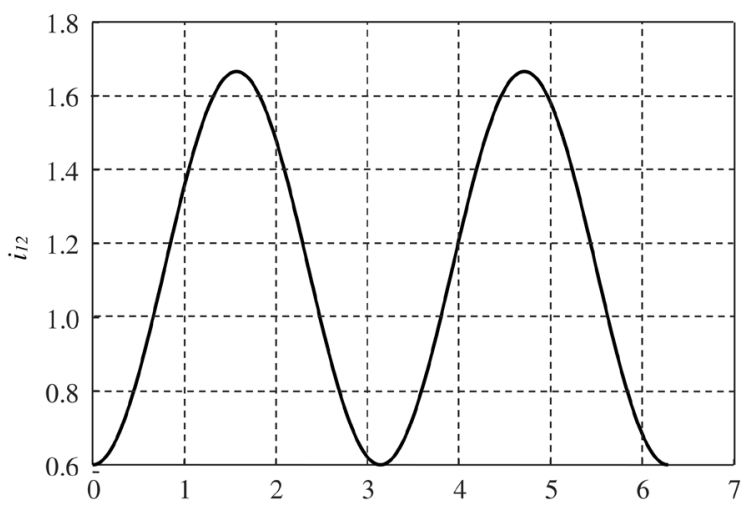

Hình 8 . Hàm tỷ số tuyền giữa hai rôto của máy nén kiểu Roots

\subsection{Thiết kế cặp BROV dẫn động rôto của máy nén}

Do cặp bánh răng dẫn động phải cùng hàm truyền với rôto nhằm đảm bảo dẫn động cặp rôto của máy nén ăn khớp đúng và không bị va đập. Do đó, thông số thiết kế đường lăn $\Sigma_{l}$ của $\mathrm{BROV}_{1}$ được chọn trùng với thông số đường lăn $\Sigma_{n 1}$ của rôto ${ }_{1}$ và với khoảng cách trục $a_{12}=80 \mathrm{~mm}$ thay vào $(6,10,20)$ và kiểm tra điều kiện cắt lẹm $(18,23)$ cũng như phân bổ số răng trên mỗi $\operatorname{BROV}(16,21,22)$. Từ đó ta tính toán số để xác định được các thông số thiết kế của thanh răng sinh và cặp $\mathrm{BROV}_{1}$ và $\mathrm{BROV}_{2}$ như được tổng hợp trong Bảng 1 và Bảng 2. Trên cơ sở đó ta có bản vẽ thiết kế được mô tả trên Hình 9 và từ Hình 9 nhận thấy các răng trên bánh răng Ô-van đều nhau về hình dạng hình học cũng như kích thước, khắc phục được hiện tượng răng không đều ở các nghiên cứu truyền thống khi sử dụng đường thân khai của đường tròn làm biên dạng răng của $\mathrm{BRKT}$ nói chung và bánh răng Ô-van nói riêng.
Bảng 1. Thông số thiết kế thanh răng sinh biên dạng xyclôít cải tiến của elíp

\begin{tabular}{|l|c|c|}
\hline \multicolumn{1}{|c|}{ Tên gọi } & $\begin{array}{c}\text { Ký } \\
\text { hiệu }\end{array}$ & Giá trị \\
\hline Bán trục lớn $\Sigma_{E}(\mathrm{~mm})$ & $a$ & 1,0 \\
\hline Bán trục nhỏ $\Sigma_{E}(\mathrm{~mm})$ & $b$ & 0,7 \\
\hline Môđun & $m$ & 3,4 \\
\hline Bước răng trên đường chia $(\mathrm{mm})$ & $p_{t}$ & 10,8 \\
\hline Chiều dày răng $(\mathrm{mm})$ & $t$ & 5,4 \\
\hline Chiều rộng rãnh răng $(\mathrm{mm})$ & $w$ & 5,4 \\
\hline Chiều cao răng (mm) & $h$ & 4,0 \\
\hline Chiều cao đỉnh răng $(\mathrm{mm})$ & $h_{a}$ & 2,0 \\
\hline Chiều cao chân răng $(\mathrm{mm})$ & $h_{f}$ & 2,0 \\
\hline
\end{tabular}

Bảng 2. Thông số thiết kế cặp BROV biên dạng xyclôít cải tiến của elíp

\begin{tabular}{|l|c|c|c|}
\hline \multicolumn{1}{|c|}{ Tên gọi } & $\begin{array}{c}\text { Ký } \\
\text { hiệu }\end{array}$ & BR 1 & $\begin{array}{c}\text { BR } \\
2\end{array}$ \\
\hline Bán trục lớn $\Sigma_{2}(\mathrm{~mm})$ & $a_{1}$ & 50,0 & - \\
\hline Bán trục nhỏ $\Sigma_{2}(\mathrm{~mm})$ & $b_{1}$ & 30,0 & - \\
\hline Môđun & $m$ & 3,4 & 3,4 \\
\hline Số răng & $z$ & 24,0 & 24,0 \\
\hline $\begin{array}{l}\text { Bước răng trên đường } \\
\text { lăn(mm) }\end{array}$ & $p$ & 10,8 & 10,8 \\
\hline Chiều dày răng $(\mathrm{mm})$ & $t$ & 5,4 & 5,4 \\
\hline Chiều rộng rãnh răng $(\mathrm{mm})$ & $w$ & 5,4 & 5,4 \\
\hline Chiều cao răng (mm) & $h$ & 4,0 & 4,0 \\
\hline Chiều cao đỉnh răng $(\mathrm{mm})$ & $h_{a}$ & 2,0 & 2,0 \\
\hline Chiều cao chân răng $(\mathrm{mm})$ & $h_{f}$ & 2,0 & 2,0 \\
\hline
\end{tabular}

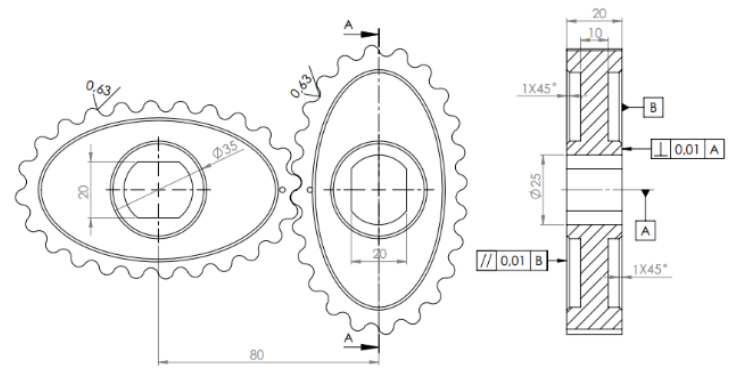

SECRONAAA

Hình 9. Bản vẽ thiết kế cặp BROV dẫn động rôto máy nén 


\subsection{Chế tạo thử nghiệm cặp BROV dẫn động máy nén}

Vật liệu chế tạo cặp BRKT là thép tấm $40 \mathrm{X}$, trước khi gia công để đảm bảo độ vuông góc giữa biên dạng răng, trục bánh răng với mặt đầu, phôi được mài phẳng hai mặt trên/dưới bằng máy mài phẳng NIPPEI TOYAMA Type SFG-30/60 (Nhật Bản) với thông số công nghệ khi mài: vận tốc trục chính $n=1800$ (vòng/phút), chiều sâu cắt $t=0,05(\mathrm{~mm})$, tốc độ tịnh tiến của phôi $v_{p h}=20$ (m/phút), lượng chạy dao dọc $\mathrm{s}_{\mathrm{d}}=30$ (m/giây). Sau đó, lô̂ trục và hai lỗ định vị $\varnothing 8 \mathrm{~mm}$ được gia công trên máy cắt dây ST3240VM (Đài Loan). Hai lỗ định vị được chế tạo nhằm mục đích định vị chính xác vị trí của bánh răng khi lật mặt gia công do chiều sâu cắt của dao không đủ. Biên dạng răng được gia công trên máy phay $\mathrm{CNC}$ HITACHI SEIKI VS40 (Nhật Bản) gồm ba nguyên công phay thô, phay bán tinh và phay tinh với các thông số công nghệ gia công được mô tả trong Bảng 3 .

Bảng 3. Thông số công nghệ gia công BROV

\begin{tabular}{|c|c|c|}
\hline $\begin{array}{l}\text { Nguyên } \\
\text { công }\end{array}$ & Thông số & Giá trị \\
\hline \multirow{4}{*}{ Phay thô } & Vận tốc trục chính & $\begin{array}{l}1500 \\
\text { (vòng/phút) }\end{array}$ \\
\hline & Chiều sâu cắt & $0,5(\mathrm{~mm})$ \\
\hline & Lượng chạy dao & 200 (mm/phút) \\
\hline & \multicolumn{2}{|c|}{$\begin{array}{l}\text { Loại dao: NACHI GRERS10-Dc10- } \\
\text { 125-L75- Ds10-N4 }\end{array}$} \\
\hline \multirow{4}{*}{$\begin{array}{l}\text { Phay bán } \\
\text { tinh }\end{array}$} & Vận tốc trục chính & $\begin{array}{l}1800 \\
\text { (vòng/phút) }\end{array}$ \\
\hline & Chiều sâu cắt & $0,2(\mathrm{~mm})$ \\
\hline & Lượng chạy dao & 150 (mm/phút) \\
\hline & \multicolumn{2}{|c|}{$\begin{array}{l}\text { Loại dao: NACHI AGHV4-Dc4-112- } \\
\text { L60- Ds8-N4 }\end{array}$} \\
\hline \multirow{4}{*}{ Phay tinh } & Vận tốc trục chính & $\begin{array}{l}3000 \\
\text { (vòng/phút) }\end{array}$ \\
\hline & Chiều sâu cắt & $0,05(\mathrm{~mm})$ \\
\hline & Lượng chạy dao & 75 (mm/phút) \\
\hline & \multicolumn{2}{|c|}{$\begin{array}{l}\text { Loại dao: NACHI AGHV4-Dc4-112- } \\
\text { L60- Ds8-N4 }\end{array}$} \\
\hline
\end{tabular}

Phương pháp gá đặt, kiểm tra, và các nguyên công gia công được mô tả trên Hình 10 , còn Hình 11 là cặp BROV sau khi chế tạo thử nghiệm.

Hình $11 \mathrm{c}$ là cặp BROV biên dạng xyclôít cải tiến của nghiên cứu này sau khi thay thế cặp BROV biên dạng thân khai. Từ Hình 9 và Hình 11 cho thấy biên dạng răng của cặp BROV sau khi được thiết kế, chế tạo theo đường xyclôít cải tiến đã khắc phục được hiện tượng răng không đều của BROV biên dạng thân khai truyền thống răng ở vị trí $I$ nhỏ hơn ở vị trí $I I$
(Hình 11b). Ngoài ra, từ Hình 11 b, c cho thấy đối với cặp BROV biên dạng xyclôít cải tiến số răng ít hơn $\mathrm{z}$ $=24$ dẫn đến kích thước và hình dạng răng to hơn so với BROV biên dạng thân khai ban đầu $\mathrm{z}=34$ răng. Điều đó làm tăng khả năng tải của bộ truyền động, trong khi BROV biên dạng thân khai không thể tăng kích thước răng bởi bị hạn chế bởi điều kiện cắt lẹm chân răng và góc áp lực thanh răng sinh.
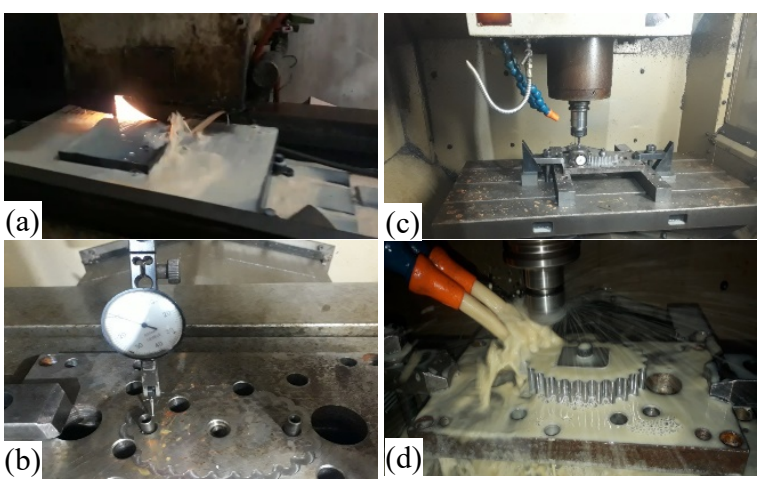

Hình 10. Các nguyên công gia công BROV với (a) nguyên công mài phẳng, (b) kiểm tra độ phẳng và vuông góc, $(\mathrm{c})$ kiểm tra giữa các nguyên công phay bán tinh và $(\mathrm{d})$ phay tinh $\mathrm{BROV}$.

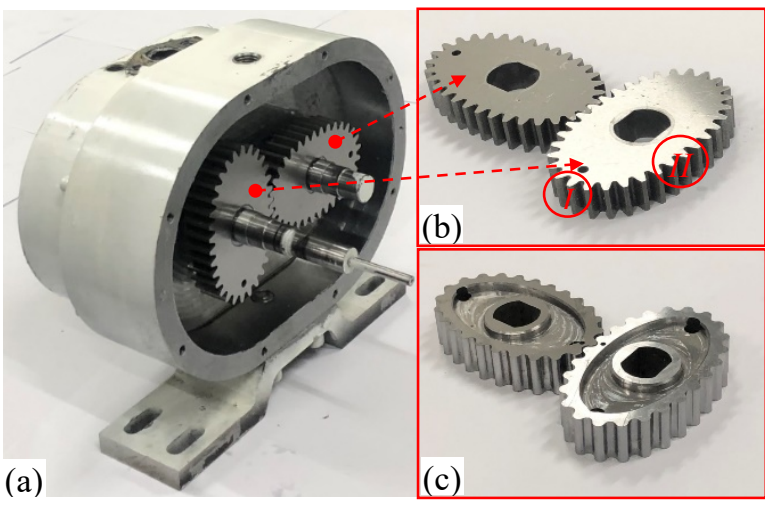

Hình 11. Ảnh chụp máy nén kiểu Roots với (a) Buồng chứa cặp bánh răng dẫn động, (b) cặp BROV biên dạng thân khai ban đầu và (c) cặp BROV biên dạng xyclôít cải tiến sau khi chế tạo thay thế

\section{Kết luận}

Từ những kết quả nghiên cứu lý thuyết và thực nghiệm, nghiên cứu này có những đóng góp cụ thể sau đây: (1) Đã thiết lập được mô hình toán học biên dạng răng của $\mathrm{BROV}$ bằng đường cong xyclôít cải tiến, với biên dạng này ta đã khắc phục được hiện tượng răng không đều ở các vị trí khác nhau trên BROV. Với kết quả nghiên cứu lý thuyết được thực hiện bởi nghiên cứu này có thể xây dựng các mô đun phần mềm tính toán thiết kế số tự động các loại BROV thông qua các điều kiện biên đã được thiết lập, (2) Với kết quả chế tạo thực nghiệm cho thấy tiềm năng của các loại BROV hoàn toán có thể được ứng dụng trong các kịch bản ứng dụng mà bánh răng trụ tròn truyền thống không thể thay thế hoặc thay thế thì phải phối hợp các bánh răng thành hệ rất phức tạp như: cơ cấu cấy trong 
máy nông nghiệp, cơ cấu gạt nước mưa ô tô, bộ biến đổi vô cấp CVT của ô tô v.V..

Tuy nhiên, việc phát triển các loại BROV với biên dạng mới được đề xuất bởi nghiên cứu này cần phải được xét về khả năng tải, tốc độ mòn v.v.. đây là những vấn đề mà chúng tôi đang tiếp tục nghiên cứu và phát triển trong tương lai.

\section{Lò̀i cảm ơn}

Nghiên cứu này được tài trợ bởi Bộ giáo dục và Đào tạo trong đề tài cấp Bộ, mã số: B2019-BKA-09. Học viên cao học Nguyễn Hoàng Việt được hỗ trợ bởi chương trình học bổng đào tạo thạc sĩ trong nước của Quỹ Đối mới sáng tạo Vingroup.

\section{Tài liệu tham khảo}

[1] Emmanuel Poulle, Équatoires et horlogerie planétaire du XIII ${ }^{\mathrm{e}}$ au XVI ${ }^{\mathrm{e}}$ siècle, Tome I, Librairie Droz, 1980, pp. $511-550$

[2] L. S. Guo, W. J. Zhang, Kinematic analysis of a rice transplanting mechanism with eccentric planetary gear trains, Mechanism and Machine Theory, vol. 36, pp. 1175 - 1188, Aug. 2001, https://doi.org/10.1016/S0094-114X(01)00052-0.

[3] X. Zhao, M. Chu, X. Ma, L. Dai, B. Ye, J. Chen, Research on design method of non-circular planetary gear train transplanting mechanism based on precise poses and trajectory optimization, AIME, vol. 10, no. 12 , pp. 1-12, Oct. 2018, https://doi.org/ 10.1177/1687814018814368.

[4] X. Zhao, J. Ye, M. Chu, L. Dai, J. Chen, Automatic scallion seedling feeding mechanism with an asymmetrical high-order transmission gear train, Chin. J. Mech. Eng., vol. 33, no. 1, pp. 2-14, Feb. 2020, https://doi.org/10.1186/s10033-020-0432-9.

[5] E. Ottaviano, D. Mundo, G. A. Danieli, M. Ceccarelli, Numerical and experimental analysis of non-circular gears and cam-follower systems as function generators, Mechanism and Machine Theory, vol. 43, pp. 996-1008, Aug. 2008, https://doi.org/10.1016/j.mechmachtheory.2007.07.00.
[6] T. Emura, A. Arakawa, A new steering mechanism using noncircular gear, JSME International Journal. Ser. 3, vol. 35, no. 4, pp. 604- 610, 1992, https://doi.org/10.1299/jsmec1988.35.604.

[7] C. F. Chen, C. B. Tsay, Computerized tooth profile generation and analysis of characteristics of elliptical gears with circular-arc teeth, Journal of Materials Processing Technology, vol. 148, no. 2, pp. 226-234 May. 2004,

https://doi.org/10.1016/j.jmatprotec.2003.07.011.

[8] F. L. Litvin, Alfonso Fuentes-Azna, Ignacio GonzalezPerez, Kenichi Hayasaka, Noncircular gears design and generation, Cambridge University Press, NY, USA, 2009.

[9] B.-W. Bair, M.-H Sung, J.-S Wang, C.-F Chen, Tooth profile generation and analysis of oval gears with circular-arc teeth, Mechanism and Machine Theory, vol. 44, no. 4, pp. 1306-1317, Jun. 2009,

https://doi.org/10.1016/j.mechmachtheory.2008.07.003

[10] X. Liu, K. Nagamura, K. Ikejo, Analysis of the dynamic characteristics of elliptical gears, JAMDSM, vol. 6, no. 4, pp. 484-497, 2012, https://doi.org/10.1299/jamdsm.6.484

[11] J. G. Li, X. Wu, S. Mao, Numerical computing method of noncircular gear tooth profiles generated by shaper cutters, Int J Adv Manuf. Technol., vol. 33, pp. 10981105, Aug. 2007,

https://doi.org/10.1007/s00170-006-0560-0

[12] B.-W. Bair, Computer aided design of non-standard elliptical gear drives, Proc. Instn. Mech. Engrs. Part C:JMES, vol. 216, no. 4, pp. 473-482, Apr. 2001, https://doi.org/10.1243/0954406021525250.

[13] T. N. Tien, N. H. Thai, N. D. Long, Effects of head and rotor clearances on leakage of flow rate and pressure in a novel non-contact Rotor blower, Vietnam Journal of Science and Technology, VJST, vol. 57, no. 4A, pp.125-140, Sep. 2019, https://doi.org/10.15625/2525-2518/57/4A/14466. 